\title{
DYNAMIC CRACK PROPAGATION BETWEEN TWO BONDED ORTHOTROPIC PLATES
}

\author{
M. S. MATBULY
}

Received 30 June 2003 and in revised form 30 August 2003

The problem of crack propagation along the interface of two bonded dissimilar orthotropic plates is considered. Using Galilean transformation, the problem is reduced to a quasistatic one. Then, using Fourier transforms and asymptotic analysis, the problem is reduced to a pair of singular integral equations with Cauchy-type singularity. These equations are solved using Gauss-Chebyshev quadrature formulae. The dynamic stress intensity factors are obtained in closed form expressions. Furthermore, a parametric study is introduced to investigate the effect of crack growth rate and geometric and elastic characteristics of the plates on values of dynamic stress intensity factors.

\section{Introduction}

Composite materials have been extensively employed in many engineering fields such as mechanical and aerospace structures. When the material used as member of such structures contains a crack, it is seriously necessary to study the stress field distribution at the immediate vicinity of crack tips. The inertia action must be considered when the applied loads or crack length depend on time. Also, the most frequently observed phenomenon in the experiments shows that the crack growth rate is constant during the extending history except in the final unstable or arresting stage [11]. So, the elastodynamic analysis of a moving crack, with constant velocity, is one of the most important problems in fracture mechanics. The dynamic stress intensity factors (DSIF) play a key role in characterizing the fracture behaviour of such problems. Thus, analytical determination of DSIF in predicting the fracture cannot be overemphasized.

In general, there are two approaches for analytical determination of DSIF. The first one employs the integral transforms and asymptotic analysis to reduce the problem to that of a system of singular integral equations $[1,3,10,15,20,22,23]$. The second approach employs complex analysis to reduce the problem to that of a system of Riemann-Hilbert problems $[12,13,14,19]$.

The present work is concerned with elastodynamic stress disturbance problem of a moving Griffith crack with constant velocity. The crack is located at the interface of two 


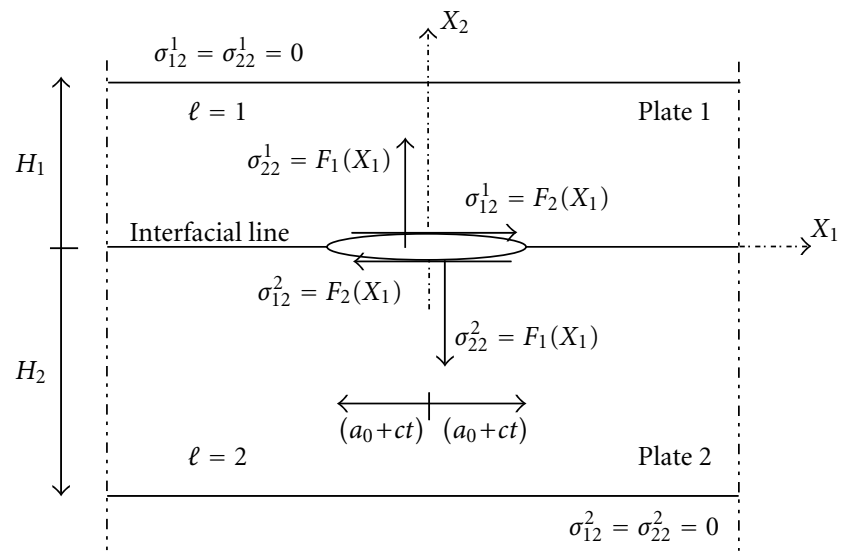

Figure 1.1. Two bonded dissimilar plates containing moving interfacial crack.

bonded dissimilar orthotropic plates, as shown in Figure 1.1. Each plate possesses a finite width and is subjected to a static stress distribution along crack surfaces. This is the main difference between the present work and previous ones $[1,3,4,6,7,8,10,12,13,14,15$, $19,20,22,23]$, which were concerned only with plates of infinite widths. The governing equations of the problem are described. Then, Fourier transforms and asymptotic analysis are employed to reduce the solution of the problem to that of a system of first-kind singular integral equations with Cauchy-type singularity. These are solved numerically according to the algorithm in [17]. Then, closed-form expressions for the asymptotic stress field distribution at the immediate vicinity of crack tips are obtained.

\section{Governing equations}

Assuming that the Cartesian coordinate axes are the axes of symmetry of the elastic materials, the displacement equations of motion for orthotropic plates are [3]

$$
\begin{aligned}
& C_{11}^{\ell} \frac{\partial^{2} U_{1}^{\ell}}{\partial X_{1}^{2}}+C_{66}^{\ell} \frac{\partial^{2} U_{1}^{\ell}}{\partial X_{2}^{2}}+\left[C_{12}^{\ell}+C_{66}^{\ell}\right] \frac{\partial^{2} U_{2}^{\ell}}{\partial X_{1} \partial X_{2}}=m^{\ell} \frac{\partial^{2} U_{1}^{\ell}}{\partial t^{2}} \\
& C_{66}^{\ell} \frac{\partial^{2} U_{2}^{\ell}}{\partial X_{1}^{2}}+C_{22}^{\ell} \frac{\partial^{2} U_{2}^{\ell}}{\partial X_{2}^{2}}+\left[C_{12}^{\ell}+C_{66}^{\ell}\right] \frac{\partial^{2} U_{1}^{\ell}}{\partial X_{1} \partial X_{2}}=m^{\ell} \frac{\partial^{2} U_{2}^{\ell}}{\partial t^{2}}
\end{aligned}
$$

where $\ell$ is a superscript ( $\ell=1$ for orthotropic material in $X_{2}>0$, while $\ell=2$ for orthotropic material in $\left.X_{2}<0\right)$, as shown in Figure $1.1 ; U_{j}^{\ell}(j=1,2)$ are the displacement components in direction of $X_{1}$ and $X_{2}$, respectively; $C_{i j}^{\ell}(i, j=1,2)$ and $C_{66}^{\ell}$ are the elastic constants of orthotropic plate materials; and $m^{\ell}$ and $t$ are the material mass density and time, respectively.

The boundary conditions along the interface of plates are

$$
\begin{aligned}
\lim _{X_{2} \rightarrow 0^{+}} \sigma_{22}^{1}\left(X_{1}, X_{2}, t\right) & =\lim _{X_{2} \rightarrow 0^{-}} \sigma_{22}^{2}\left(X_{1}, X_{2}, t\right)=F_{1}\left(X_{1}\right), \\
\lim _{X_{2} \rightarrow 0^{+}} \sigma_{12}^{1}\left(X_{1}, X_{2}, t\right) & =\lim _{X_{2} \rightarrow 0^{-}} \sigma_{12}^{2}\left(X_{1}, X_{2}, t\right)=F_{2}\left(X_{1}\right),
\end{aligned}
$$




$$
\begin{aligned}
& \lim _{X_{2} \rightarrow 0^{+}} U_{1}^{1}\left(X_{1}, X_{2}, t\right)=\lim _{X_{2} \rightarrow 0^{-}} U_{1}^{2}\left(X_{1}, X_{2}, t\right), \\
& \lim _{X_{2} \rightarrow 0^{+}} U_{2}^{1}\left(X_{1}, X_{2}, t\right)=\lim _{X_{2} \rightarrow 0^{-}} U_{2}^{2}\left(X_{1}, X_{2}, t\right), \quad\left|X_{1}\right|>a_{0}+d \\
& \lim _{X_{2} \rightarrow 0^{+}} \sigma_{22}^{1}\left(X_{1}, X_{2}, t\right)=\lim _{X_{2} \rightarrow 0^{-}} \sigma_{22}^{2}\left(X_{1}, X_{2}, t\right), \\
& \lim _{X_{2} \rightarrow 0^{+}} \sigma_{12}^{1}\left(X_{1}, X_{2}, t\right)=\lim _{X_{2} \rightarrow 0^{-}} \sigma_{12}^{2}\left(X_{1}, X_{2}, t\right), \quad\left|X_{1}\right|>a_{0}+d,
\end{aligned}
$$

where $a_{0}$ is the initial half crack length, and $d=|c| t$, where $c$ is the magnitude of crack propagation velocity. Moreover, $F_{i}\left(X_{1}\right)(i=1,2)$ are known functions. They represent the applied static stress along crack surfaces; $F_{1}\left(-X_{1}\right)=F_{1}\left(X_{1}\right)$ and $F_{2}\left(-X_{1}\right)=-f_{2}\left(-X_{1}\right)$.

The boundary conditions along the external boundaries are

$$
\begin{aligned}
\sigma_{12}^{1}\left(X_{1}, H_{1}, t\right) & =\sigma_{22}^{1}\left(X_{1}, H_{1}, t\right)=\sigma_{12}^{2}\left(X_{1},-H_{2}, t\right) \\
& =\sigma_{22}^{2}\left(X_{1},-H_{2}, t\right)=0, \quad\left|X_{1}\right|<\infty,
\end{aligned}
$$

where $H_{1}$ and $H_{2}$ are as shown in Figure 1.1.

Using Galilean transformation: $x=\left(X_{1}-c t\right) / a_{0}, y=X_{2} / a_{0}$, and $t=t$, the governing equations (2.1), (2.2), and (2.3) can be reduced to a quasistatic form as follows:

$$
\begin{aligned}
& \frac{\partial^{2} u^{\ell}}{\partial x^{2}}+a_{1}^{\ell} \frac{\partial^{2} v^{\ell}}{\partial x \partial y}+a_{2}^{\ell} \frac{\partial^{2} u^{\ell}}{\partial y^{2}}=0, \\
& \frac{\partial^{2} v^{\ell}}{\partial x^{2}}+b_{1}^{\ell} \frac{\partial^{2} u^{\ell}}{\partial x \partial y}+b_{2}^{\ell} \frac{\partial^{2} v^{\ell}}{\partial y^{2}}=0,
\end{aligned} \quad(\ell=1,2)
$$

where

$$
\begin{aligned}
u^{\ell}(x, y) & =\frac{U_{1}^{\ell}\left(X_{1}, X_{2}, t\right)}{a_{0}}, & v^{\ell}(x, y) & =\frac{U_{2}^{\ell}\left(X_{1}, X_{2}, t\right)}{a_{0}}, \\
a_{1}^{\ell} & =\frac{C_{12}^{\ell}+C_{66}^{\ell}}{C_{11}^{\ell}\left[1-\left(M_{1}^{\ell}\right)^{2}\right]}, & a_{2}^{\ell} & =\frac{C_{66}^{\ell}}{C_{11}^{\ell}\left[1-\left(M_{1}^{\ell}\right)^{2}\right]}, \\
b_{1}^{\ell} & =\frac{C_{12}^{\ell}+C_{66}^{\ell}}{C_{66}^{\ell}\left[1-\left(M_{2}^{\ell}\right)^{2}\right]}, & b_{2}^{\ell} & =\frac{C_{22}^{\ell}}{C_{66}^{\ell}\left[1-\left(M_{2}^{\ell}\right)^{2}\right]}, \\
M_{j}^{\ell} & =\frac{c}{V_{j}^{\ell}}(j=1,2), & V_{1}^{\ell} & =\sqrt{\frac{C_{11}^{\ell}}{m^{\ell}}}, \quad V_{2}^{\ell}=\sqrt{\frac{C_{66}^{\ell}}{m^{\ell}}}
\end{aligned}
$$

Furthermore, the values of Mach numbers $M_{j}^{\ell}(\ell, j=1,2)$ are assumed to be less than unity for subsonic crack propagation. 
The boundary conditions for the reduced quasistatic problem can be expressed as follows:

$$
\begin{aligned}
& \lim _{y \rightarrow 0^{+}} \sigma_{y y}^{1}(x, y)=\lim _{y \rightarrow 0^{-}} \sigma_{y y}^{2}(x, y)=f_{1}(x), \\
& \lim _{y \rightarrow 0^{+}} \sigma_{x y}^{1}(x, y)=\lim _{y \rightarrow 0^{-}} \sigma_{x y}^{2}(x, y)=f_{2}(x), \quad|x|<1, \\
& \lim _{y \rightarrow 0^{+}} u^{1}(x, y)=\lim _{y \rightarrow 0^{-}} u^{2}(x, y), \\
& \lim _{y \rightarrow 0^{+}} \nu^{1}(x, y)=\lim _{y \rightarrow 0^{-}} v^{2}(x, y), \quad|x|>1, \\
& \lim _{y \rightarrow 0^{+}} \sigma_{y y}^{1}(x, y)=\lim _{y \rightarrow 0^{-}} \sigma_{y y}^{2}(x, y), \\
& \lim _{y \rightarrow 0^{+}} \sigma_{x y}^{1}(x, y)=\lim _{y \rightarrow 0^{-}} \sigma_{x y}^{2}(x, y), \quad|x|>1, \\
& \sigma_{y y}^{1}\left(x, h_{1}\right)=\sigma_{x y}^{1}\left(x, h_{1}\right)=\sigma_{y y}^{2}\left(x,-h_{2}\right)=\sigma_{x y}^{2}\left(x,-h_{2}\right)=0, \quad|x|<\infty,
\end{aligned}
$$

where

$$
\begin{gathered}
\sigma_{x y}^{\ell}=\frac{\sigma_{12}^{\ell}}{Q}, \quad \sigma_{y y}^{\ell}=\frac{\sigma_{22}^{\ell}}{Q}, \quad Q=\sqrt{C_{66}^{1} C_{66}^{2}}, \\
h_{\ell}=\frac{H_{\ell}}{a_{0}} \quad(\ell=1,2) .
\end{gathered}
$$

\section{Solution of the problem}

Decoupling (2.4) then employing Fourier sine (cosine) transforms with respect to $x$, one can find that

$$
\begin{aligned}
& u^{\ell}(x, y)=\frac{1}{\pi} \sum_{j=1}^{4}\left(\int_{0}^{\infty} A_{j}^{\ell}(\alpha) e^{\alpha r_{j}^{\ell} y} \sin \alpha x d \alpha\right), \quad(\ell=1,2) \\
& \nu^{\ell}(x, y)=\frac{1}{\pi} \sum_{j=1}^{4}\left(\int_{0}^{\infty} k_{j}^{\ell} A_{j}^{\ell}(\alpha) e^{\alpha r_{j}^{\ell} y} \cos \alpha x d \alpha\right),
\end{aligned}
$$

where $\alpha$ is the transform variable, $A_{j}^{\ell}(\alpha)(\ell=1,2$ and $j=1,4)$ are unknown functions, and $r_{j}^{\ell}(\ell=1,2$ and $j=1,4)$ are the real distinct roots of

$$
f_{1}^{\ell} r^{4}-2 f_{2}^{\ell} r^{2}+1=0 \quad(\ell=1,2)
$$

provided that

$$
\begin{aligned}
& f_{1}^{\ell}=a_{2}^{\ell} b_{2}^{\ell}, \quad 2 f_{2}^{\ell}=a_{2}^{\ell}+b_{2}^{\ell}-a_{1}^{\ell} b_{1}^{\ell}, \quad f_{2}^{\ell}>\sqrt{f_{1}^{\ell}}, f_{1}^{\ell}>0, \\
& k_{j}^{\ell}=\frac{a_{2}^{\ell}\left(r_{j}^{\ell}\right)^{2}-1}{a_{1}^{\ell} r_{j}^{\ell}} \quad(\ell=1,2, j=1,4) .
\end{aligned}
$$


From the stress-displacement relationship of orthotropic materials [9], one can get

$$
\begin{aligned}
\sigma_{x y}^{\ell}(x, y) & =\frac{C_{66}^{\ell}}{Q}\left[\frac{\partial v^{\ell}}{\partial x}+\frac{\partial u^{\ell}}{\partial y}\right] \\
& =\frac{1}{\pi} \sum_{j=1}^{4}\left(\int_{0}^{\infty} \alpha p_{j}^{\ell} A_{j}^{\ell}(\alpha) e^{\alpha r_{j}^{\ell} y} \sin \alpha x d \alpha\right) \quad(\ell=1,2), \\
\sigma_{y y}^{\ell}(x, y) & =\frac{1}{Q}\left[C_{12}^{\ell} \frac{\partial u^{\ell}}{\partial x}+C_{22}^{\ell} \frac{\partial \nu^{\ell}}{\partial y}\right] \\
& =\frac{1}{\pi} \sum_{j=1}^{4}\left(\int_{0}^{\infty} \alpha o_{j}^{\ell} A_{j}^{\ell}(\alpha) e^{\alpha r_{j}^{\ell} y} \cos \alpha x d \alpha\right) \quad(\ell=1,2),
\end{aligned}
$$

where

$$
p_{j}^{\ell}=\frac{C_{66}^{\ell}\left\lfloor r_{j}^{\ell}-k_{j}^{\ell}\right\rfloor}{Q}, \quad o_{j}^{\ell}=\frac{\left\lfloor C_{12}^{\ell}+C_{22}^{\ell} r_{j}^{\ell} k_{j}^{\ell}\right\rfloor}{Q}
$$

On suitable substitution from (2.9) into (3.5), one can find that

$$
A_{m}^{\ell}(\alpha)=\sum_{j=3}^{4} L_{j m}^{\ell} e^{\alpha \beta_{j m}^{\ell}} A_{j}^{\ell}(\alpha) \quad(m, \ell=1,2),
$$

where

$$
\begin{aligned}
& \beta_{j m}^{\ell}=\left\{\begin{array}{ll}
h_{1}\left(r_{j}^{\ell}-r_{m}^{\ell}\right) & \text { for } \ell=1 \\
h_{2}\left(r_{m}^{\ell}-r_{j}^{\ell}\right) & \text { for } \ell=2
\end{array} \quad(m=1,2, j=3,4),\right. \\
& L_{j m}^{\ell}=\frac{\sum_{n=1}^{2}(-1)^{n}\left(1-\delta_{n m}\right)\left[p_{n}^{\ell} o_{j}^{\ell}-p_{j}^{\ell} o_{n}^{\ell}\right]}{p_{1}^{\ell} o_{2}^{\ell}-p_{2}^{\ell} o_{1}^{\ell}} \quad(\ell, m=1,2, j=3,4),
\end{aligned}
$$

$\delta_{n m}$ is the Kronecker delta.

Substituting (3.5) and (3.7) into (2.8), one can find that

$$
\begin{aligned}
& A_{3}^{2}(\alpha)=\frac{E_{4}^{2} D_{3}^{1}-D_{4}^{2} E_{3}^{1}}{\Delta} A_{3}^{1}(\alpha)+\frac{E_{4}^{2} D_{4}^{1}-D_{4}^{2} E_{4}^{1}}{\Delta} A_{4}^{1}(\alpha), \\
& A_{4}^{2}(\alpha)=\frac{D_{3}^{2} E_{3}^{1}-E_{3}^{2} D_{3}^{1}}{\Delta} A_{3}^{1}(\alpha)+\frac{D_{3}^{2} E_{4}^{1}-E_{3}^{2} D_{4}^{1}}{\Delta} A_{4}^{1}(\alpha),
\end{aligned}
$$

where

$$
\begin{gathered}
\Delta=D_{3}^{2} E_{4}^{2}-D_{4}^{2} E_{3}^{2}, \\
D_{j}^{\ell}=p_{j}^{\ell}+\sum_{m=1}^{2} p_{m}^{\ell} L_{j m}^{\ell} e^{\alpha \beta_{j m}^{\ell}}, \quad E_{j}^{\ell}=o_{j}^{\ell}+\sum_{m=1}^{2} o_{m}^{\ell} L_{j m}^{\ell} e^{\alpha \beta_{j m}^{\ell}} \quad(\ell=1,2, j=3,4) .
\end{gathered}
$$


The boundary conditions (2.6) and (2.7) in conjunction with (3.7), (3.8), (3.9), (3.10), and (3.11) lead to

$$
\begin{array}{ll}
\frac{1}{\pi} \int_{0}^{\infty} \alpha\left[E_{3}^{1} A_{3}^{1}(\alpha)+E_{4}^{1} A_{4}^{1}(\alpha)\right] \cos \alpha x d \alpha=f_{1}(x), & |x|<1, \\
\frac{1}{\pi} \int_{0}^{\infty} \alpha\left[D_{3}^{1} A_{3}^{1}(\alpha)+D_{4}^{1} A_{4}^{1}(\alpha)\right] \sin \alpha x d \alpha=f_{2}(x), & \\
\frac{1}{\pi} \int_{0}^{\infty} \alpha\left[\tau_{1}(\alpha) A_{3}^{1}(\alpha)+\tau_{2}(\alpha) A_{4}^{1}(\alpha)\right] \sin \alpha x d \alpha=0, & \\
\frac{1}{\pi} \int_{0}^{\infty} \alpha\left[\xi_{1}(\alpha) A_{3}^{1}(\alpha)+\xi_{2}(\alpha) A_{4}^{1}(\alpha)\right] \cos \alpha x d \alpha=0, & |x|>1,
\end{array}
$$

where

$$
\begin{aligned}
\tau_{1}(\alpha)= & {\left[1+\sum_{m=1}^{2} L_{3 m}^{1} e^{\alpha \beta_{3 m}^{1}}-\left(1+\sum_{m=1}^{2} L_{3 m}^{2} e^{\alpha \beta_{3 m}^{2}}\right) \Delta_{1}-\left(1+\sum_{m=1}^{2} L_{4 m}^{2} e^{\alpha \beta_{4 m}^{2}}\right) \Delta_{2}\right], } \\
\tau_{2}(\alpha)= & {\left[1+\sum_{m=1}^{2} L_{4 m}^{1} e^{\alpha \beta_{4 m}^{1}}-\left(1+\sum_{m=1}^{2} L_{3 m}^{2} e^{\alpha \beta_{3 m}^{2}}\right) \Delta_{3}-\left(1+\sum_{m=1}^{2} L_{4 m}^{2} e^{\alpha \beta_{4 m}^{2}}\right) \Delta_{4}\right], } \\
\xi_{1}(\alpha)= & {\left[k_{3}^{1}+\sum_{m=1}^{2} k_{m}^{1} L_{3 m}^{1} e^{\alpha \beta_{3 m}^{1}}-\left(k_{3}^{2}+\sum_{m=1}^{2} k_{m}^{2} L_{3 m}^{2} e^{\alpha \beta_{3 m}^{2}}\right) \Delta_{1}\right.} \\
& \left.-\left(k_{4}^{2}+\sum_{m=1}^{2} k_{m}^{2} L_{4 m}^{2} e^{\alpha \beta_{4 m}^{2}}\right) \Delta_{2}\right] \\
\xi_{2}(\alpha)= & {\left[k_{4}^{1}+\sum_{m=1}^{2} k_{m}^{1} L_{4 m}^{1} e^{\alpha \beta_{4 m}^{1}}-\left(k_{3}^{2}+\sum_{m=1}^{2} k_{m}^{2} L_{3 m}^{2} e^{\alpha \beta_{3 m}^{2}}\right) \Delta_{3}\right.} \\
& \left.-\left(k_{4}^{2}+\sum_{m=1}^{2} k_{m}^{2} L_{4 m}^{2} e^{\alpha \beta_{4 m}^{2}}\right) \Delta_{4}\right] \\
\Delta_{1}= & \frac{E_{4}^{2} D_{3}^{1}-D_{4}^{2} E_{3}^{1}}{\Delta}, \quad \Delta_{2}=\frac{D_{3}^{2} E_{3}^{1}-E_{3}^{2} D_{3}^{1}}{\Delta}, \\
\Delta_{3}= & \frac{E_{4}^{2} D_{4}^{1}-D_{4}^{2} E_{4}^{1}}{\Delta}, \quad \Delta_{4}=\frac{D_{3}^{2} E_{4}^{1}-E_{3}^{2} D_{4}^{1}}{\Delta} .
\end{aligned}
$$

The unknown functions $A_{j}^{1}(\alpha)(j=3,4)$ can be determined through solving the integral equations (3.12) and (3.13) as follows [21].

Let

$$
\begin{aligned}
& \lim _{y \rightarrow 0^{+}} \frac{\partial \nu^{1}(x, y)}{\partial x}-\lim _{y \rightarrow 0^{-}} \frac{\partial \nu^{2}(x, y)}{\partial x}=\phi_{1}(x)[1-H(x-1)] \\
& \lim _{y \rightarrow 0^{+}} \frac{\partial u^{1}(x, y)}{\partial x}-\lim _{y \rightarrow 0^{-}} \frac{\partial u^{2}(x, y)}{\partial x}=\phi_{2}(x)[1-H(x-1)]
\end{aligned}
$$

where $H(x-1)$ is the unit step function [2], while $\phi_{j}(x)(j=1,2)$ are unknown odd and even functions of $x$, respectively. 
From (3.13) and (3.15), one can deduce that

$$
\begin{aligned}
& A_{3}^{1}(\alpha)=\frac{\tau_{2} \Phi_{1}(\alpha)+\xi_{2} \Phi_{2}(\alpha)}{(\alpha \Psi)}, \\
& A_{4}^{1}(\alpha)=\frac{-\left[\tau_{1} \Phi_{1}(\alpha)+\xi_{1} \Phi_{2}(\alpha)\right]}{(\alpha \Psi)}
\end{aligned}
$$

where

$$
\begin{aligned}
\Phi_{1}(\alpha) & =2 \int_{0}^{1} \phi_{1}(x) \sin \alpha x d x, \\
\Phi_{2}(\alpha) & =2 \int_{0}^{1} \phi_{2}(x) \cos \alpha x d x, \\
\Psi & =\tau_{1} \xi_{2}-\tau_{2} \xi_{1} .
\end{aligned}
$$

Substituting (3.16) into (3.12), the problem is reduced to the following system of integral equations:

$$
\sum_{j=1}^{2} \int_{-1}^{1} \breve{H}_{i j}(x, t) \phi_{j}(t) d t=f_{i}(x), \quad|x|<1, i=1,2
$$

where the kernels $\breve{H}_{i j}(x, t)$ are

$$
\begin{aligned}
& \breve{H}_{11}(x, t)=\frac{1}{\pi} \int_{0}^{\infty} \frac{1}{\Psi}\left[E_{3}^{1} \tau_{2}-E_{4}^{1} \tau_{1}\right] \sin \alpha(t-x) d \alpha, \\
& \breve{H}_{12}(x, t)=\frac{1}{\pi} \int_{0}^{\infty} \frac{1}{\Psi}\left[E_{3}^{1} \xi_{2}-E_{4}^{1} \xi_{1}\right] \cos \alpha(t-x) d \alpha, \\
& \breve{H}_{21}(x, t)=\frac{1}{\pi} \int_{0}^{\infty} \frac{1}{\Psi}\left[D_{3}^{1} \tau_{2}-D_{4}^{1} \tau_{1}\right] \cos \alpha(t-x) d \alpha, \\
& \breve{H}_{22}(x, t)=-\frac{1}{\pi} \int_{0}^{\infty} \frac{1}{\Psi}\left[D_{3}^{1} \xi_{2}-D_{4}^{1} \xi_{1}\right] \sin \alpha(t-x) d \alpha .
\end{aligned}
$$

Since the integrands of (3.19) are continuous functions of $\alpha$, then it is clear that any possible singularity of the kernels must result from the asymptotic analysis of the integrands as $t \rightarrow x$ and $\alpha \rightarrow \infty[16,21]$. Then, by adding and subtracting the asymptotic expressions of these integrands under the integral sign, the problem can be reduced to the following pair of singular integral equations with Cauchy-type singularity:

$$
\frac{1}{\pi} \sum_{j=1}^{2}\left(\delta_{i j} G_{i j} \int_{-1}^{1} \frac{\phi_{j}(t)}{t-x} d t+\int_{-1}^{1} H_{i j}(x, t) \phi_{j}(t) d t\right)=f_{i}(x), \quad|x|<1, i=1,2
$$


where

$$
\begin{aligned}
& H_{11}(x, t)=\int_{0}^{\infty}\left\langle\frac{1}{\Psi}\left[E_{3}^{1} \tau_{2}-E_{4}^{1} \tau_{1}\right]-G_{11}\right\rangle \sin \alpha(t-x) d \alpha, \\
& H_{12}(x, t)=\int_{0}^{\infty}\left\langle\frac{1}{\Psi}\left[E_{3}^{1} \xi_{2}-E_{4}^{1} \xi_{1}\right]-G_{12}\right\rangle \cos \alpha(t-x) d \alpha, \\
& H_{21}(x, t)=\int_{0}^{\infty}\left\langle\frac{1}{\Psi}\left[D_{3}^{1} \tau_{2}-D_{4}^{1} \tau_{1}\right]-G_{21}\right\rangle \cos \alpha(t-x) d \alpha, \\
& H_{22}(x, t)=\int_{0}^{\infty}\left\langle\frac{-1}{\Psi}\left[D_{3}^{1} \xi_{2}-D_{4}^{1} \xi_{1}\right]-G_{22}\right\rangle \sin \alpha(t-x) d \alpha, \\
& G_{11}=\frac{\left\langle o_{3}^{1}\left(B-s_{2}\right) / B-o_{4}^{1}\left(B-s_{1}\right) / B\right\rangle}{Z}, \\
& G_{12}=\frac{\left\langle o_{3}^{1}\left(k_{4}^{1} B-s_{4}\right) / B-o_{4}^{1}\left(k_{3}^{1} B-s_{3}\right) / B\right\rangle}{Z}, \\
& G_{21}=\frac{\left\langle p_{3}^{1}\left(B-s_{2}\right) / B-p_{4}^{1}\left(B-s_{1}\right) / B\right\rangle}{Z}, \\
& G_{22}=\frac{\left\langle p_{4}^{1}\left(k_{3}^{1} B-s_{3}\right) / B-p_{3}^{1}\left(k_{4}^{1} B-s_{4}\right) / B\right\rangle}{Z}, \\
& Z=\left(k_{4}^{1}-k_{3}^{1}\right)+\frac{s_{3}-s_{4}+k_{3}^{1} s_{2}-k_{4}^{1} s_{1}}{B}+\frac{s_{1} s_{4}-s_{2} s_{3}}{B^{2}}, \\
& B=\left(L_{31}^{2} L_{42}^{2}-L_{41}^{2} L_{32}^{2}\right)\left(p_{1}^{2} o_{2}^{2}-p_{2}^{2} o_{1}^{2}\right), \\
& s_{1}=\left(B_{13}+B_{24}\right) L_{31}^{2} L_{42}^{2}+\left(B_{12}+B_{27}\right) L_{41}^{2} L_{32}^{2} \text {, } \\
& s_{2}=\left(B_{33}+B_{44}\right) L_{31}^{2} L_{42}^{2}+\left(B_{32}+B_{47}\right) L_{41}^{2} L_{32}^{2} \text {, } \\
& s_{3}=\left(k_{1}^{2} B_{13}+k_{2}^{2} B_{24}\right) L_{31}^{2} L_{42}^{2}+\left(k_{2}^{2} B_{12}+k_{1}^{2} B_{27}\right) L_{41}^{2} L_{32}^{2} \text {, } \\
& s_{4}=\left(k_{1}^{2} B_{33}+k_{2}^{2} B_{44}\right) L_{31}^{2} L_{42}^{2}+\left(k_{2}^{2} B_{32}+k_{1}^{2} B_{47}\right) L_{41}^{2} L_{32}^{2} \text {, } \\
& B_{13}=p_{3}^{1} o_{2}^{2}-p_{2}^{2} o_{3}^{1}, \quad B_{24}=p_{1}^{2} o_{3}^{1}-p_{3}^{1} o_{1}^{2}, \\
& B_{12}=p_{3}^{1} o_{1}^{2}-p_{1}^{2} o_{3}^{1}, \quad B_{27}=p_{2}^{2} o_{3}^{1}-p_{3}^{1} o_{2}^{2}, \\
& B_{33}=p_{4}^{1} o_{2}^{2}-p_{2}^{2} o_{4}^{1}, \quad B_{44}=p_{1}^{2} o_{4}^{1}-p_{4}^{1} o_{1}^{2}, \\
& B_{32}=p_{4}^{1} o_{1}^{2}-p_{1}^{2} o_{4}^{1}, \quad B_{47}=p_{2}^{2} o_{4}^{1}-p_{4}^{1} o_{2}^{2} \text {. }
\end{aligned}
$$

From (3.15) and (2.5d), one can deduce the single-valuedness conditions ensuring the uniqueness of $\phi_{i}(\alpha)(i=1,2)$ as follows:

$$
\int_{-1}^{1} \phi_{1}(t) d t=0, \quad \int_{-1}^{1} \phi_{2}(t) d t=\frac{2}{c}\left(\sqrt{\frac{C_{11}^{2}}{m^{2}}}-\sqrt{\frac{C_{11}^{1}}{m^{1}}}\right) .
$$

Equations (3.20) and (3.22) can be solved as follows [17]. 
Assume that

$$
\phi_{i}(t)=\frac{q_{i}(t)}{\sqrt{1-t^{2}}} \quad(i=1,2)
$$

where $q_{i}(t)(i=1,2)$ are bounded continuous functions for all $t \in[-1,1]$.

By substituting from (3.23) into (3.20) and (3.22) then employing Gauss-Chebyshev integration formulae, the solution of the problem can be reduced to the following system of linear algebraic equations [17]:

$$
\begin{gathered}
\sum_{j=1}^{2} \sum_{k=1}^{n_{1}}\left(w_{k}\left[\frac{\delta_{i j} G_{i j}}{t_{k}-x_{l}}+H_{i j}\left(x_{l}, t_{k}\right)\right] q_{j}\left(t_{k}\right)\right)=f_{i}\left(x_{l}\right) \quad(i=1,2)\left(x_{l}=1, n_{1}-1\right), \\
\sum_{k=1}^{n_{1}} w_{k} q_{1}\left(t_{k}\right)=0, \\
\sum_{k=1}^{n_{1}} w_{k} q_{2}\left(t_{k}\right)=\frac{2}{\pi c}\left(\sqrt{\frac{C_{11}^{2}}{m^{2}}}-\sqrt{\frac{C_{11}^{1}}{m^{1}}}\right),
\end{gathered}
$$

where $n_{1}$ is the number of collocation points in the interval $[-1,1]$,

$$
\begin{gathered}
w_{1}=w_{n_{1}}=\frac{1}{2 n_{1}-2}, \quad w_{k}=\frac{1}{n_{1}-1} \quad\left(\text { for } k=2, n_{1}-1\right) \\
t_{k}=\cos \left(\frac{\pi(k-1)}{n_{1}-1}\right) \quad\left(k=1, n_{1}\right) \\
x_{l}=\cos \left(\frac{\pi(2 l-1)}{2 n_{1}-2}\right) \quad\left(l=1, n_{1}-1\right) .
\end{gathered}
$$

For the concerned problem, one can deduce that the DSIF at the left and right crack tips are equal. Then, by making use of the following asymptotic relations, as $\alpha \rightarrow \infty$, [5]:

$$
\begin{gathered}
\Phi_{1}(\alpha)=\int_{-1}^{1} \frac{q_{1}(t)}{\sqrt{1-t^{2}}} \sin \alpha t d t \approx q_{1}(1) \sqrt{\frac{2 \pi}{\alpha}} \sin \left(\alpha-\frac{\pi}{4}\right)+\vartheta\left(\frac{1}{\alpha}\right), \\
\Phi_{2}(\alpha)=\int_{-1}^{1} \frac{q_{2}(t)}{\sqrt{1-t^{2}}} \cos \alpha t d t \approx q_{2}(1) \sqrt{\frac{2 \pi}{\alpha}} \cos \left(\alpha-\frac{\pi}{4}\right)+\vartheta\left(\frac{1}{\alpha}\right), \\
\int_{0}^{\infty} \frac{1}{\sqrt{\alpha}} e^{-b \alpha}\left\{\begin{array}{l}
\sin h \alpha \\
\cosh \alpha
\end{array}\right\} d \alpha=\frac{\sqrt{\pi}}{\left(b^{2}+h^{2}\right)^{0.25}}\left\{\begin{array}{l}
\sin \\
\cos
\end{array}\right\}\left(0.5 \tan ^{-1}\left(\frac{h}{b}\right)\right), \quad b>0,
\end{gathered}
$$


the leading terms of the asymptotic stress field distribution (3.5) at the immediate vicinity of the right crack tip $\left(x \rightarrow 1\right.$ and $\left.y \rightarrow 0^{ \pm}\right)$can be obtained as follows:

$$
\begin{aligned}
\sigma_{x y}(x, y) \approx & q_{1}(1)\left(\frac{\zeta_{1}}{\sqrt{2 \rho_{1}}} \sin \frac{\theta_{1}}{2}-\frac{\zeta_{2}}{\sqrt{2 \rho_{2}}} \sin \frac{\theta_{2}}{2}\right) \\
& +q_{2}(1)\left(\frac{\zeta_{3}}{\sqrt{2 \rho_{1}}} \cos \frac{\theta_{1}}{2}-\frac{\zeta_{4}}{\sqrt{2 \rho_{2}}} \cos \frac{\theta_{2}}{2}\right), \\
\sigma_{y y}(x, y) \approx & q_{1}(1)\left(\frac{\gamma_{1}}{\sqrt{2 \rho_{1}}} \cos \frac{\theta_{1}}{2}-\frac{\gamma_{2}}{\sqrt{2 \rho_{2}}} \cos \frac{\theta_{2}}{2}\right) \\
& +q_{2}(1)\left(\frac{\gamma_{3}}{\sqrt{2 \rho_{1}}} \sin \frac{\theta_{1}}{2}-\frac{\gamma_{4}}{\sqrt{2 \rho_{2}}} \sin \frac{\theta_{2}}{2}\right),
\end{aligned}
$$

where

$$
\begin{array}{ll}
\rho_{1}=\sqrt{(x-1)^{2}+\left(r_{3}^{1} y\right)^{2},} & \rho_{2}=\sqrt{(x-1)^{2}+\left(r_{4}^{1} y\right)^{2},} \\
\theta_{1}=\tan ^{-1}\left(\frac{r_{3}^{1} y}{x-1}\right), & \theta_{2}=\tan ^{-1}\left(\frac{r_{4}^{1} y}{x-1}\right), \\
\xi_{1}=\frac{p_{3}^{1}\left(1-s_{2} / B\right)}{Z}, & \xi_{2}=\frac{p_{4}^{1}\left(1-s_{1} / B\right)}{Z}, \\
\xi_{3}=\frac{p_{3}^{1}\left(k_{4}^{1}-s_{4} / B\right)}{Z}, & \xi_{4}=\frac{p_{4}^{1}\left(k_{3}^{1}-s_{3} / B\right)}{Z}, \\
\gamma_{1}=\frac{o_{3}^{1}\left(1-s_{2} / B\right)}{Z}, & \gamma_{2}=\frac{o_{4}^{1}\left(1-s_{1} / B\right)}{Z}, \\
\gamma_{3}=\frac{o_{3}^{1}\left(k_{4}^{1}-s_{4} / B\right)}{Z}, & \gamma_{4}=\frac{o_{4}^{1}\left(k_{3}^{1}-s_{3} / B\right)}{Z} .
\end{array}
$$

Therefore, the DSIF, $K_{I}$, and $K_{I I}$ can be determined as follows [18]:

$$
K_{I}+i K_{I I}=Q \sqrt{2 a_{0}} \lim _{x \rightarrow 1, y \rightarrow 0^{+}} \sqrt{x-1}\left[\sigma_{y y}(x, y)+i \sigma_{x y}(x, y)\right]
$$

such that by substituting from (3.27) and (3.28) into (3.29) then evaluating the limits, one can find that

$$
\begin{aligned}
K_{I} & =Q \sqrt{a_{0}}\left[\gamma_{1}-\gamma_{2}\right] q_{1}(1), \\
K_{I I} & =Q \sqrt{a_{0}}\left[\xi_{3}-\xi_{4}\right] q_{2}(1) .
\end{aligned}
$$




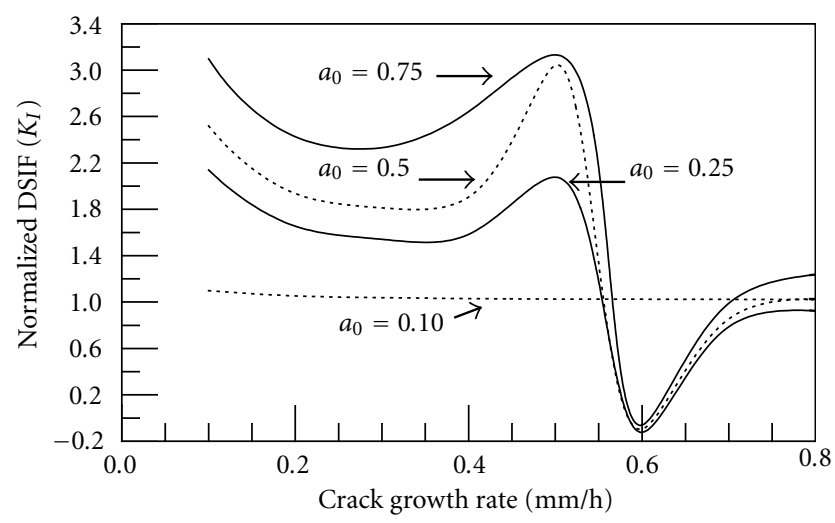

Figure 4.1. Variation of the normalized $K_{I}$ with the crack growth rate and initial crack length.

\section{Numerical results}

A parametric study is introduced to investigate the effects of crack growth rate, initial crack length, and the plate width on the values of DSIF. Consider that a structure of two bonded orthotropic plates as in Figure 1.1 possesses the following elastic characteristics:

$$
\begin{gathered}
m^{1}=m^{2}=1 \mathrm{~g} / \mathrm{cm}^{3}, \\
C_{66}^{1}=C_{66}^{2}=1 \mathrm{MPa}, \\
C_{22}^{1}=2.5 \mathrm{MPa}, \\
C_{22}^{2}=4 \mathrm{MPa}, \\
C_{11}^{\ell}=\left(1+r_{1}^{\ell}\right) C_{66}^{\ell}-r_{1}^{\ell}\left(r_{2}^{\ell}\right)^{2} C_{22}^{\ell} \quad(\ell=1,2), \\
C_{12}^{\ell}=\left[\sqrt{r_{1}^{\ell}\left[\left(r_{2}^{\ell}\right)^{2}+1\right]-\left[\left(r_{1}^{\ell}\right)^{2}+\left(r_{2}^{\ell}\right)^{2}\right]}-1\right] C_{66}^{\ell} \quad(\ell=1,2),
\end{gathered}
$$

where (4.1) are derived from (2.5), (3.2), and (3.3). Also, for the concerned numerical results, it is assumed that $1>r_{1}^{\ell}>r_{2}^{\ell}>0, r_{3}^{\ell}=-r_{1}^{\ell}$, and $r_{4}^{\ell}=-r_{2}^{\ell}$. The initial crack length $2 a_{0}$ ranges from 0.2 to $1.5 \mathrm{~cm}$, while the width of plates ranges from 5 to $10 \mathrm{~m}$. For simplicity, the numerical results are obtained for constant uniform stress distribution along the crack surfaces, $|x|<1$ and $y \rightarrow \pm 0$.

Furthermore, we have found that $\left|\int_{0}^{\infty} I_{i j}(\alpha, x, t) d \alpha-\int_{0}^{4} I_{i j}(\alpha, x, t) d \alpha\right|<10^{-16}$, where $I_{i, j}(\alpha, x, t)(i, j=1,2)$, are the integrands of (3.21). Therefore, the improper integrals of (3.21) are approximated and evaluated numerically from $\alpha=0 \rightarrow 4$ by using the trapezoidal rule. Then, the values of DSIF are normalized such that

$$
\text { Normalized } K_{I}=\frac{K_{I}}{\sigma_{22}^{1} \sqrt{a_{0}}}, \quad \text { Normalized } K_{I I}=\frac{K_{I I}}{\sigma_{12}^{1} \sqrt{a_{0}}},
$$

where $\sigma_{22}^{1}$ and $\sigma_{12}^{1}$ represent the applied stress along the upper crack surface. 


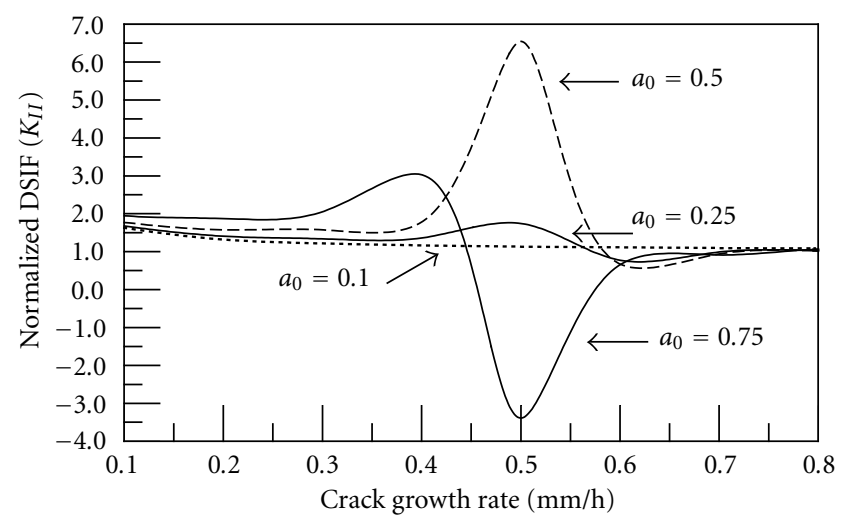

Figure 4.2. Variation of the normalized $K_{I I}$ with the crack growth rate and initial crack length.

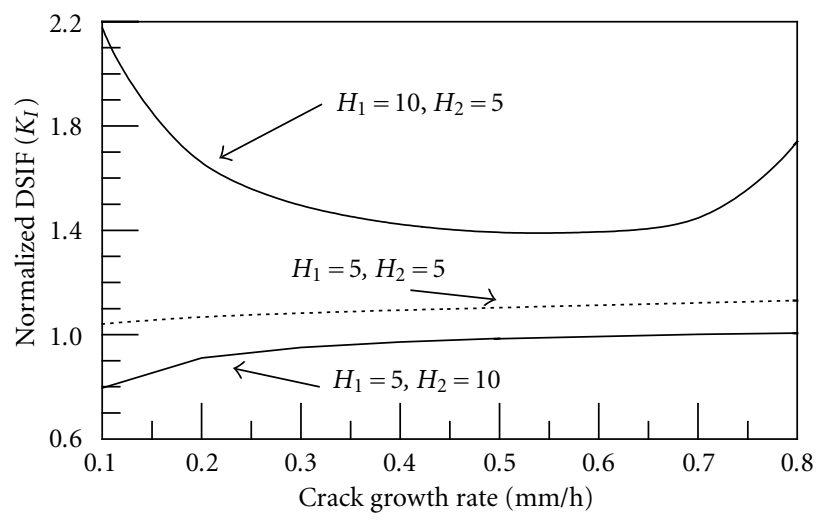

Figure 4.3. Variation of the normalized $K_{I}$ with the crack growth rate for different plate widths.

Figure 4.1 shows that the value of normalized $K_{I}$ continuously decreases with increasing the crack growth rate, where $0<c<0.32$ and $0.5<c<0.6$, while it increases elsewhere. Also, it shows increasing the pathological oscillatory behaviour [3] for the normalized $K_{I}$ with increasing the initial crack length.

Figure 4.2 shows that the value of normalized $K_{I I}$ continuously increases with increasing the crack growth rate except for $0.5<c<0.65$. But this decreasing interval is shifted to $0.4<c<0.5$ for the case of a relatively long initial crack length $2 a_{0}=1.5 \mathrm{~cm}$. One can notice as well that the pathological oscillatory behaviour of the normalized $K_{I I}$ increases with increasing $a_{0}$.

For the prescribed elastic and geometric characteristics, Figure 4.3 shows that the value of normalized $K_{I}$ continuously increases with increasing the crack growth rate except when $c<0.5$ and $H_{1}>H_{2}$, it is decreased. Also, Figure 4.4 shows that the value of normalized $K_{I I}$ continuously decreases with increasing the crack growth rate, while it increases with increasing $c$ when $c>0.62$ and $H_{1}<H_{2}$. 


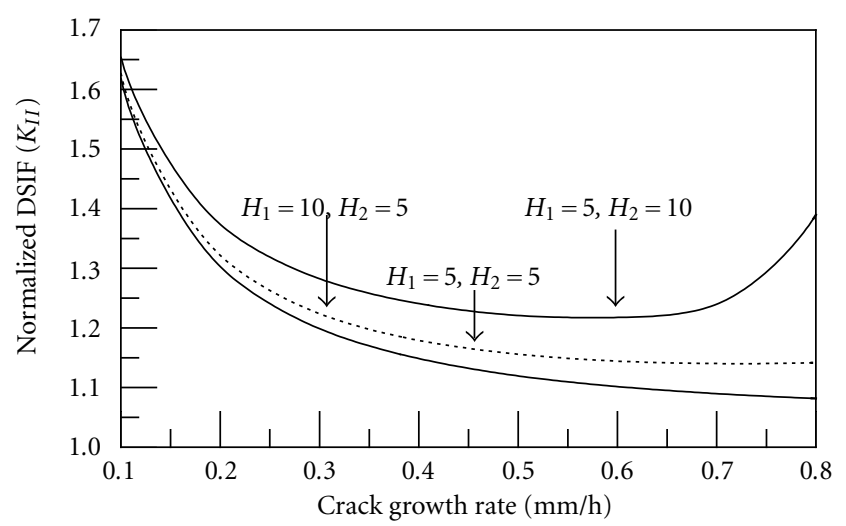

Figure 4.4. Variation of the normalized $K_{I I}$ with the crack growth rate for different plate widths.

\section{Conclusion}

The present work is concerned with elastodynamic analysis of crack propagation between two bonded dissimilar orthotropic plates. The width of each plate is assumed to be finite. This is the new trend and the main difference between this work and the previous ones $[1,3,4,6,7,8,10,12,13,14,15,19,20,22,23]$. So, the present work can be considered as an extension for the analysis of interfacial crack problems.

\section{References}

[1] R. Babaei and S. A. Lukasiewicz, Dynamic response of a crack in a functionally graded material between two dissimilar half planes under antiplane shear impact, Engrg. Fracture Mech. 60 (1998), 479-487.

[2] R. N. Bracewell, The Fourier Transform and Its Applications, McGraw-Hill Series in Electrical Engineering, Circuits and Systems, McGraw-Hill, New York, 1986.

[3] S. Das and B. Patra, Moving Griffith crack at the interface of two dissimilar orthotropic half planes, Engrg. Fracture Mech. 54 (1996), 523-531.

[4] - Stress intensity factors for moving interfacial crack between bonded dissimilar fixed orthotropic layers, Comput. \& Structures 69 (1998), no. 4, 459-472.

[5] F. Delale and F. Erdogan, Transverse shear effect in a circumferentially cracked cylindrical shell, Quart. Appl. Math. 37 (1979), 239-258.

[6] A. H. England, A crack between dissimilar media, Trans. ASME J. Appl. Mech. 32 (1965), 400402.

[7] F. Erdogan and G. Gupta, Layered composites with an interface flow, Internat. J. Solids Structures 7 (1971), 1089-1107.

[8] F. Erdogan and B. Wu, Interface crack problems in layered orthotropic materials, J. Mech. Phys. Solids 41 (1993), no. 5, 889-917.

[9] R. F. Gibson, Principles of Composite Material Mechanics, McGraw-Hill, New York, 1994.

[10] S. Itou, Dynamic stress intensity factors around two rectangular cracks in a half-space under impact load, Acta Mech. 121 (1997), no. 1-4, 153-164.

[11] J. F. Kalthoff, J. Beinert, and S. Winkler, Measurements of dynamic stress intensity factors for fast running cracks in double-cantilever-beam specimens, Fast Fracture and Crack Arrest (G. T. Hahn and M. F. Kanninen, eds.), ASTM STP, vol. 627, American Society for Testing and Materials, Pennsylvania, 1977, pp. 161-176. 
[12] K. H. Lee, Stress and displacement fields for propagating the crack along the interface of dissimilar orthotropic materials under dynamic mode I and mode II load, Trans. ASME J. Appl. Mech. 67 (2000), 223-228.

[13] K. H. Lee, J. S. Hawong, and S. H. Choi, Dynamic stress intensity factors $K_{I}, K_{I I}$ and dynamic crack propagation characteristics of orthotropic material, Engrg. Fracture Mech. 53 (1996), 119-140.

[14] J. Y. Liou and J. C. Sung, Singularities at the tip of a crack terminating normally at an interface between two orthotropic media, Trans. ASME J. Appl. Mech. 63 (1996), 264-270.

[15] C.-C. Ma and Y.-S. Ing, Dynamic crack propagation in a layered medium under antiplane shear, Trans. ASME J. Appl. Mech. 64 (1997), no. 1, 66-72.

[16] M. S. Matbuly, Mode II stress intensity factor for cracked layered material, The 3rd International Conference on Engineering Mathematics and Physics (Cairo, Egypt), Faculty of Engineering, Cairo University, Cairo, 1997, pp. 801-808.

[17] M. S. Matbuly, S. A. Mohamed, and M. Nassar, On the numerical solution of the first kind singular integral equations, The 3rd International Conference on Engineering Mathematics and Physics (Cairo, Egypt), Faculty of Engineering, Cairo University, Cairo, 1997, pp. 11-17.

[18] V. Z. Parton and E. M. Morozov, Elastic-Plastic Fracture Mechanics, Mir Publishers, Moscow, 1978.

[19] J. R. Rice, Elastic fracture mechanics concepts for interfacial cracks, Trans. ASME J. Appl. Mech. 55 (1988), 98-103.

[20] C. Rubio-Gonzalez and J. J. Mason, Response of finite cracks in orthotropic materials due to concentrated impact shear loads, Trans. ASME J. Appl. Mech. 66 (1999), no. 2, 485-491.

[21] N. I. Shbeeb, W. K. Binienda, and K. L. Kreider, Analysis of the driving forces for multiple cracks in an infinite non-homogeneous plate, part I: theoretical analysis, Trans. ASME J. Appl. Mech. 66 (1999), no. 2, 492-500.

[22] M. Valentini, S. K. Serkov, D. Bigoni, and A. B. Movchan, Crack propagation in a brittle elastic material with defects, Trans. ASME J. Appl. Mech. 66 (1999), 79-86.

[23] B. L. Wang, J. C. Han, and S. Y. Du, Dynamic response for non-homogeneous piezoelectric medium with multiple cracks, Engrg. Fracture Mech. 61 (1998), 607-617.

M. S. Matbuly: Department of Engineering Mathematics and Physics, Faculty of Engineering, Zagazig University, P.O. 44519, Zagazig, Egypt

E-mail address: mohamedmatbuly@hotmail.com 


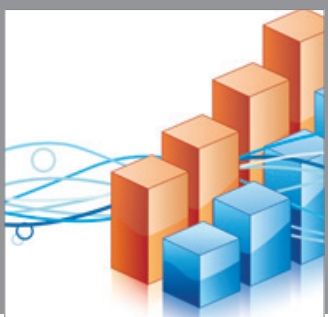

Advances in

Operations Research

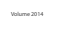

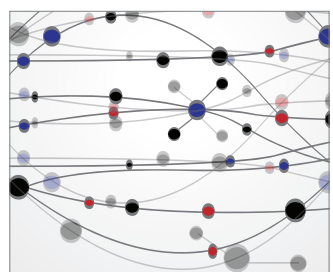

\section{The Scientific} World Journal
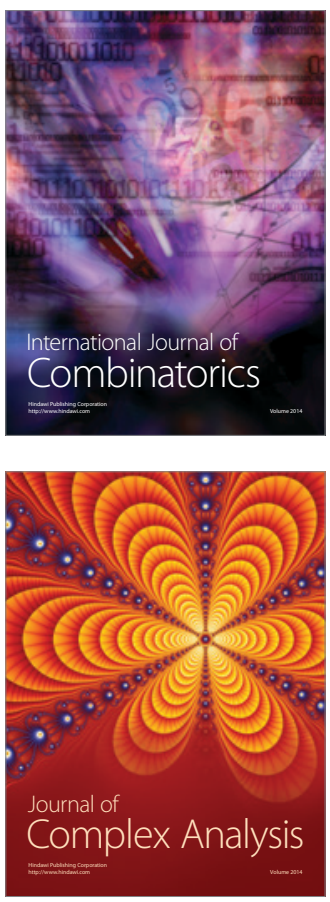

International Journal of

Mathematics and

Mathematical

Sciences
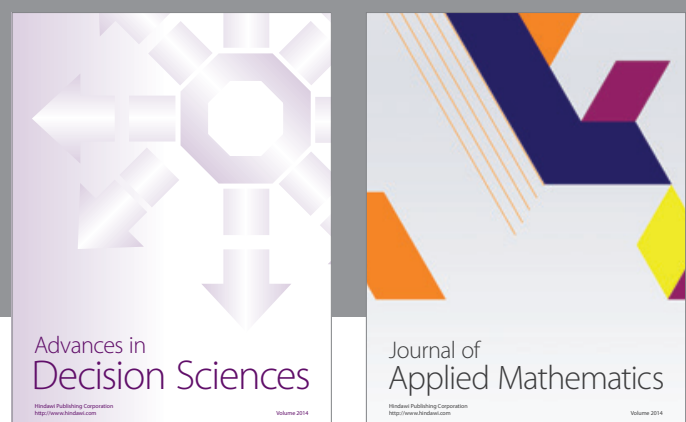

Journal of

Applied Mathematics
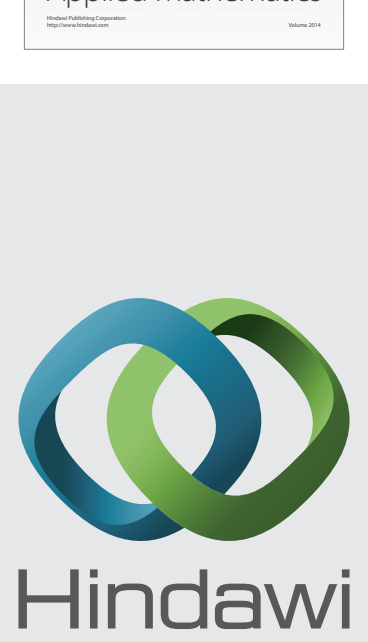

Submit your manuscripts at http://www.hindawi.com
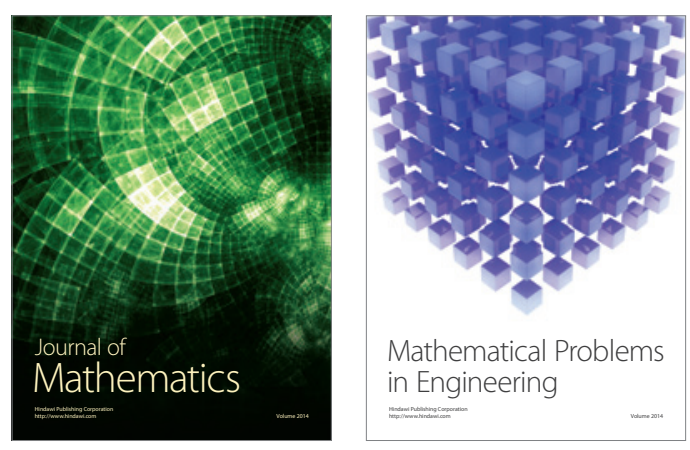

Mathematical Problems in Engineering
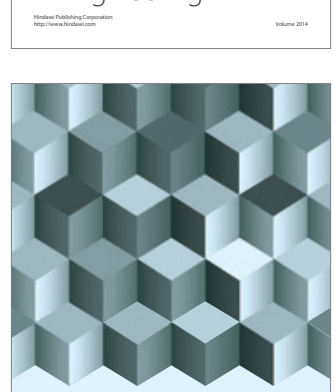

Journal of

Function Spaces
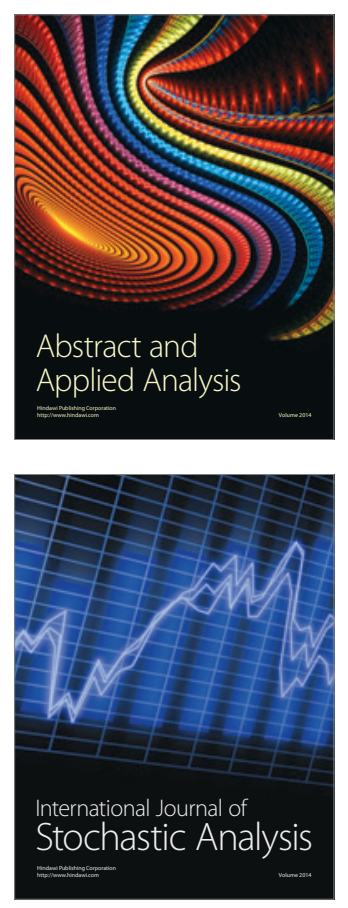

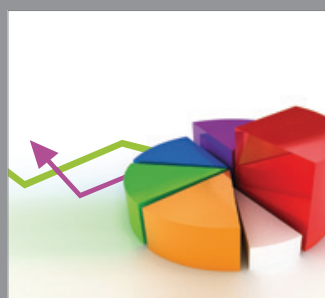

ournal of

Probability and Statistics

Promensencen
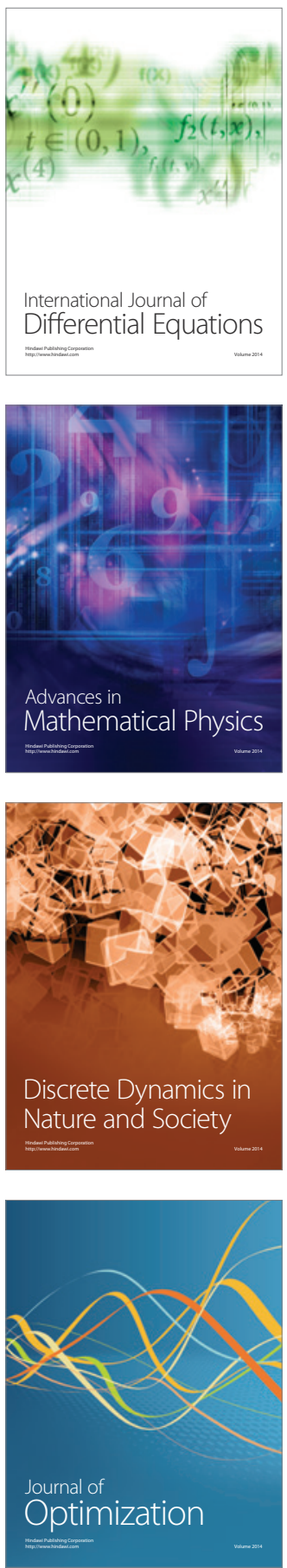